\title{
Rate of Capillary Rise in the Porous Media under Microgravity
}

\author{
Yuichi KANEKI, Yasushi SASAKI, Kuniyoshi ISHII and Manabu IGUCHI
}

Div. of Materials Science and Engineering, Graduate School of Engineering, Hokkaido University, Sapporo, Japan 060-8628

The capillary flow rate through the porous glass media under microgravity was measured by using the drop-shaft type microgravity facility of Japan Microgravity Center. Based on a simple model, the rate of the capillary rise rate of liquids was developed and expressed by

$$
\begin{aligned}
& \mathrm{t}=\left(8 \mu / \mathrm{a}^{2} \rho \mathrm{g}\right)\left(\mathrm{h}_{0} \ln \left(\mathrm{h}_{0} /\left(\mathrm{h}_{0}-\mathrm{h}\right)\right)-\mathrm{h}\right) \\
& \mathrm{h}_{0}=2 \sigma \cos \theta / \mathrm{a} \rho \mathrm{g}
\end{aligned}
$$

where $t$ is time, $\mu$ is a viscosity coefficient, a is a capillary radius, $\rho$ is a density, $g$ is the gravitational constant, $h$ is a capillary rise distance and $h_{0}$ is the equilibrium rise distance.

The behavior of the capillary rise in the porous media under microgravity is reasonably explained by the developed equation. But, the capillary rise along the vertical direction under normal gravity did not agree with the equations, and this disagreement was discussed based on the dynamic contact angle. It is estimated that the dynamic contact angle under $\mu \mathrm{G}$ will be different from that under $1 \mathrm{G}$.

KEY WORDS: capillary flow; porous media; microgravity; wetting; dynamic contact angle.

\section{Introduction}

The presence of surface tension on the free surface of a liquid sometimes affects the motion of the liquid, especially in two cases: when the interface has the finite curvature, and when the surface tension varies from point to point in the liquid surface. Typical phenomenon for the first case is a capillary rise $^{1-5)}$, and the latter case is a Marangoni convection. The Marangoni convection has been widely studied due to its importance in a chemical engineering field. Compared with the studies of Marangoni convection, the capillary rise phenomena has not attracted the wide attentions despite of its importance.

The capillary rise of liquids is, however, often encountered in practice and in nature. It plays an important role in wetting phenomena or liquid motions through porous media, especially under microgravity. Because the capillary rise phenomena that were suppressed under normal gravity became dominant under microgravity. For example, many troubles in the experiments under microgravity were taken place due to the unexpected effects of capillary rise. Therefore, understanding the capillary rise phenomena under microgravity condition is very important.

Most of studies on the capillary rise have been carried out under equilibrium conditions. A few works are dynamic ones. To understand the detail of the capillary rise phenomena, it is necessary to study not only the static behavior but also the dynamic one.

To investigate the effect of microgravity for the dynamics of the capillary rise, the capillary flow rate through a porous media under microgravity was measured. The measurements of capillary rise rates under microgravity were carried out by using the drop-shaft type microgravity facility of Japan Microgravity Center (JAMIC).

\section{Experimental Setup}

Experimental arrangement is illustrated in Fig.1. It is mainly consisted of two acrylic resin boxes. One was for a holder of a porous glass plate (40 x $50 \times 15 \mathrm{~mm})$, and another was used for an ethanol reservoir $(40 \times 90 \times 15 \mathrm{~mm})$. The porous glass plate was separated from an acrylic resin container box by using a few small acrylic resin separators. The average pore diameter of the glass porous plate is about $0.1 \mathrm{~mm}$. The two boxes were connected with a small acrylic resin tube and ethanol was able to move from the reservoir to the porous glass plate through the acrylic resin tube during experiments. The one end of the acrylic resin tube was just located below the bottom of the porous plate.

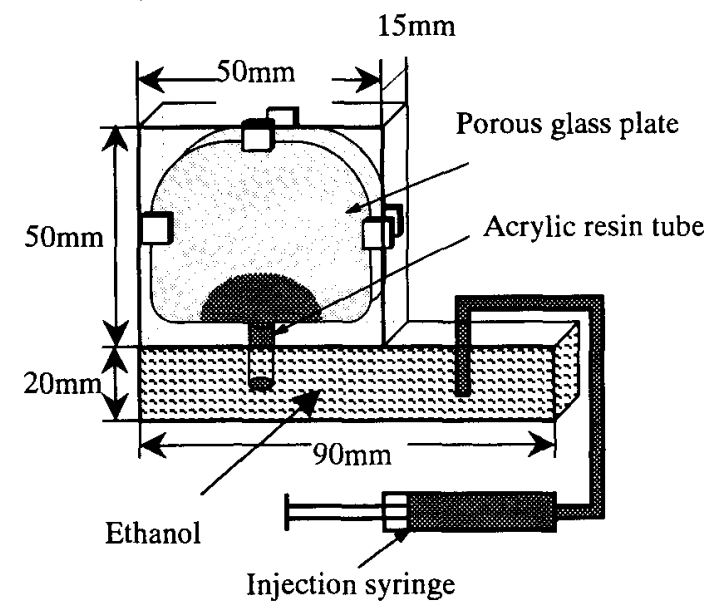

Fig.1 Experimental arrangement

Initially the ethanol reservoir was filled with ethanol. Just after the free fall, ethanol was introduced through the acrylic resin tube $(8 \mathrm{~mm}$ OD) to the porous glass plate with 
a constant rate by using a syringe and a stepping motor. The inside diameter of the acrylic resin tube was made slightly larger than the thickness of porous glass plate, and the some amount of the supplied ethanol was always allowed to spilled-out from the tube to avoid the insufficient ethanol supply. The spilled-out ethanol was able to return to the reservoir. The supply rate of ethanol was determined by the pre-experiment.

The capillary rise rate of ethanol in the porous glass plate was recorded by using a video camera during the free fall. To clearly visualize a spreading flow pattern, the ethanol was red-colored by an acid red indicator $\left(\mathrm{C}_{27} \mathrm{H}_{29} \mathrm{~N}_{2} \mathrm{NaO}_{7} \mathrm{~S}_{2}\right)$. The amount of added indicator was too small to influence the physical properties of ethanol.

The measurements of the capillary rise rate under microgravity were carried out by using the drop-shaft type microgravity facility of JAMIC. The total path length of free fall is $490 \mathrm{~m}$ and the duration of free fall is about 10 seconds. During the free fall, a microgravity environment of less than $5 \times 10^{-4} \mathrm{G}\left(\mathrm{G}=9.8 \mathrm{~m} / \mathrm{s}^{2}\right)$ is created continuously. As a reference, the capillary rise heights under normal gravity were also measured by using the same apparatus.

\section{Rate of Capillary Raise Model}

In order to evaluate the capillary rise phenomena in the porous glass plate, a simple model is adapted. Namely, as a first approximation, the porous glass plate is regarded as a bundle of many capillary tubes and a capillary rise rate in a particular direction is assumed to be represented by that of a single capillary tube in a particular direction. The capillary rise model and coordinate system are shown in Fig.2. The model considers a cylindrical liquid column in a capillary tube.

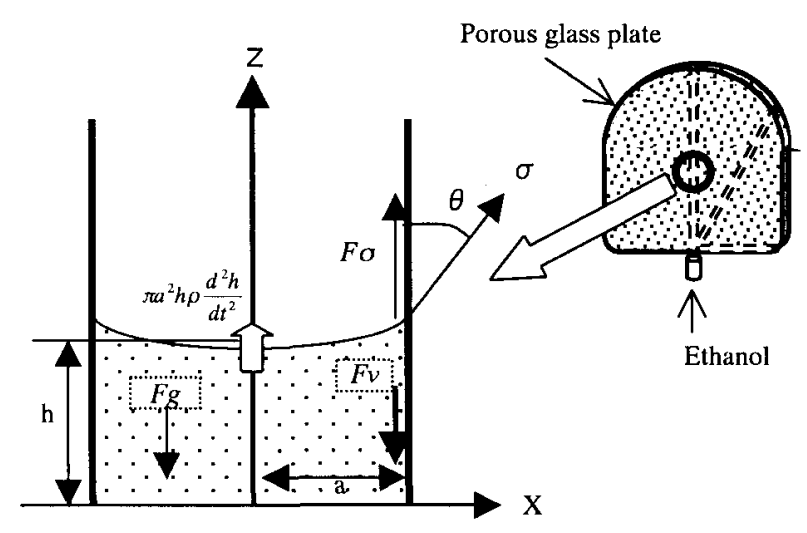

Fig.2 The capillary rise model and the coordinate system.

The equation of capillary rise motion is given from the momentum balance,

$$
\pi a^{2} h \rho\left(d^{2} h / d t^{2}\right)=F_{\sigma}-F_{g}-F_{v}
$$

The each term $F_{o}, F_{g}$ and $F_{v}$ is a force due to a surface tension, a gravitational force on the ethanol column and a viscous force on the capillary tube. The term a is a radius of capillary tube and $h$ is a height of the capillary rise. Each force can be expressed by

$$
F_{g}=\pi a^{2} h \rho g
$$

$$
\begin{aligned}
& \mathrm{F}_{\sigma}=2 \pi \mathrm{aocos} \theta \\
& \mathrm{F}_{v}=2 \pi \mathrm{ah} \tau_{\mathrm{w}}
\end{aligned}
$$

where $\rho$ is a density of liquid, $\sigma$ is a surface tension, and $\theta$ is a contact angle, $g$ is a gravitational constant and $\tau_{w}$ is a shear force. Generally, a shear force $\tau_{w}$ under unsteady state is approximately expressed ${ }^{6)}$ by

$$
\tau_{\mathrm{w}}=(8 \mu / 2 \mathrm{a}) \mathrm{dh} / \mathrm{dt}+(2 \mathrm{a} \rho / 12) \mathrm{d}^{2} \mathrm{~h} / \mathrm{dt}^{2}
$$

where the 1 st term represents the viscous resistance and the 2nd term is the resistance force proportional to the acceleration. Then,

$$
F_{v}=(8 \pi h \mu) d h / d t+\left(\pi a^{2} \rho h / 3\right) d^{2} h / d t^{2}
$$

Combined (1) to (4) and (6) yields,

$$
(4 / 3) d^{2} h / d t^{2}+\left(8 v / a^{2}\right) d h / d t=2 \sigma \cos \theta / a h \rho-g
$$

where $\nu$ is $\mu / \rho$. In this experiment, the capillary rise was actually terminated within about 4 second. Comparing the order of the 1 st term and the 2 nd term in eq. (7),

$\left((4 / 3) \mathrm{d}^{2} \mathrm{~h} / \mathrm{dt}^{2}\right) /\left(\left(8 \mu / \mathrm{a}^{2} \rho\right) \mathrm{dh} / \mathrm{dt}\right) \approx\left(4 \mathrm{~h} / 3 \mathrm{t}^{2}\right) /\left(8 \mathrm{vh} / \mathrm{a}^{2} \mathrm{t}\right) \approx \mathrm{a}^{2} / 6 v \mathrm{t}$

Substituting the values of $\mathrm{a}=0.01 \mathrm{~cm}, v=0.015 \mathrm{~cm}^{2} / \mathrm{s}$ and $\mathrm{t}$ $=4 \mathrm{~s}$, we find

$$
\mathrm{a}^{2} / 6 v \mathrm{t} \approx 0.0003
$$

Namely, the 2 nd term in eq.(7) can be negligible. Then, eq.(7) will be simplified to

$$
(8 \mu / \mathrm{a} \rho) \mathrm{dh} / \mathrm{dt}=2 \sigma \cos \theta / \mathrm{ah} \rho-\mathrm{g}
$$

Integrating eq.(8), we obtain,

$$
\mathrm{t}=\left(8 \mu / \mathrm{a}^{2} \rho \mathrm{g}\right)\left(\mathrm{h}_{0} \ln \left(\mathrm{h}_{0} /\left(\mathrm{h}_{0}-\mathrm{h}\right)\right)-\mathrm{h}\right)
$$

where $h_{0}$ is an equilibrium height of capillary rise, and is defined by

$$
2 \sigma \cos \theta / a-\rho g h_{0}=0
$$

Apparently the same equation was deduced by Levich ${ }^{6)}$ assuming the Poiseuille flow and the capillary pressure of $2 \sigma / a$. Similar consideration for the case of a horizontal capillary leads to the following expression for the time to travel a distance $l$ along the capillary

$$
\mathrm{t}=2 \mu l^{2} / \mathrm{a} \sigma \cos \theta
$$

Namely, in the case of the capillary flow in the horizontal direction under normal gravity, the capillary flow distance is proportional to $\sqrt{ } t$.

\section{Results and Discussion}

The observed spreading flow patterns of ethanol in the porous glass plate under $5 \times 10^{-4} \mathrm{G}$ and normal gravity are shown in Fig.3.

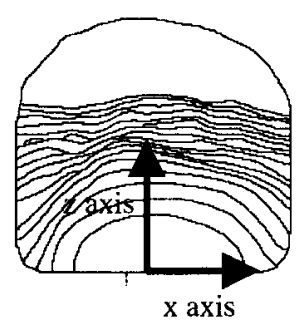

Normal gravity (1G)

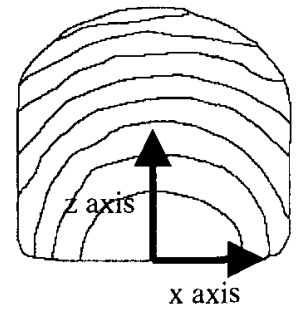

Microgravity $\left(5 \times 10^{-4} \mathrm{G}\right)$
Fig.3 The spreading flow patterns of ethanol in the porous glass plate measured under normal gravity and $10^{-4} \mathrm{G}$. 
Each contour line represents the front line of the spreading flow for every 0.5 second. The flow under $5 \times 10^{-4} \mathrm{G}$ spreads almost concentrically from the area where ethanol was supplied. Under normal gravity condition, however, ethanol spreads much faster in the horizontal direction than the vertical direction even only after 0.5 second.

From these spreading flow patterns, the spreading distance in the vertical and horizontal directions under $5 \times 10^{-4} \mathrm{G}$ and $1 \mathrm{G}$ conditions is plotted as a function of time, and are shown in Fig. 4 and Fig.5.

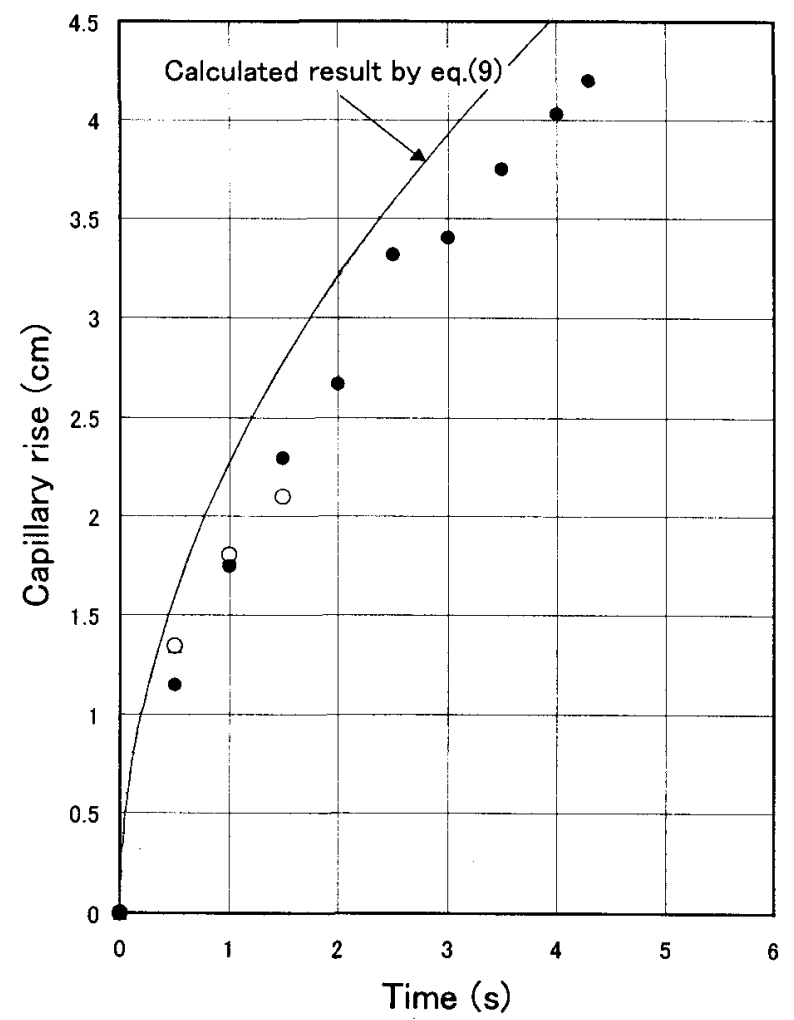

Fig.4 The capillary rise rate in the vertical and horizontal direction under microgravity as a function of time. Open circles are the horizontal direction and closed circles for the vertical direction.

Calculated lines based on the eqs.(9) and (10) are also shown in Fig.4 and 5. The calculated capillary rise values by eq. (10) is essentially the same as that obtained by eq. (9) for the initial 10 seconds.

The values of the physical properties ${ }^{8)}$ and constants used for the calculations are shown in Table 1.

Table 1 Physical properties of ethanol

\begin{tabular}{|lccl|}
\hline $\begin{array}{l}\text { Density } \\
\left(\mathrm{Kg} / \mathrm{m}^{3}\right)\end{array}$ & $\begin{array}{c}\text { viscosity } \\
(\mathrm{Pas})\end{array}$ & $\begin{array}{c}\text { surface tension } \\
(\mathrm{mN} / \mathrm{m})\end{array}$ & $\begin{array}{l}\text { contact angle } \\
(\text { degree })\end{array}$ \\
\hline $0.791 \times 10^{-3}$ & 22.27 & $1.082 \times 10^{-3}$ & 0 \\
\hline
\end{tabular}

The capillary rise under microgravity and the horizontal direction under normal gravity are reasonably explained by the eqs. (9) and (10). But, the capillary rise distance in the vertical direction under normal gravity did not agree with the eq. (9).

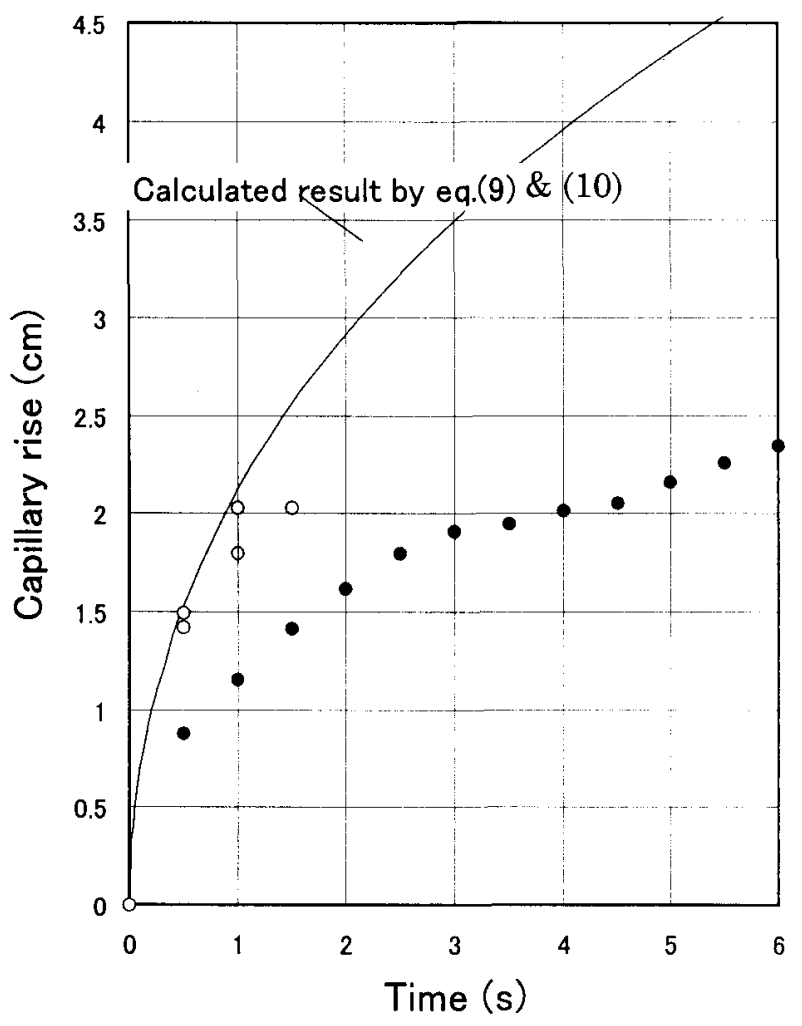

Fig.5 The capillary rise rate in the vertical and horizontal directions under normal gravity as a function of time. Open circles are the horizontal direction and closed circles for the vertical direction.

Due to the gravitational force, ethanol may tend to flow to the horizontal direction under normal gravity condition. If so, the capillary flow rate in the horizontal direction can be faster than that calculated from the eq. (9). However, as already shown, it is reasonably agreed with the calculated ones. Therefore, the disagreement of capillary flow under normal gravity is not explained by the tendency to flow to the horizontal direction.

In the present calculation of the capillary rise, the contact angle of zero for ethanol is used. This value was obtained under equilibrium condition. It is well known, however, that the contact angle under equilibrium condition is different from that in dynamic conditions, i.e., the so-called dynamic contact angle ${ }^{1-5)}$. And also as presented schematically in Fig.6, the dynamic contact angle under $\mu \mathrm{G}$ will not vary along the contact line since the curvature of surface can be determined only by the surface tension. But under normal 
gravity condition, the dynamic contact angle in the inclined tube vary along the contact line because the curvature of surface is determined by the balance between the surface tension and the gravitational force.

Therefore, it could be reasonable that the dynamic contact angle during the capillary rise under microgravity and normal gravity may not be zero, and also the dynamic contact angle under normal gravity condition will be different from that under $\mu$ gravity ${ }^{\text {). }}$.

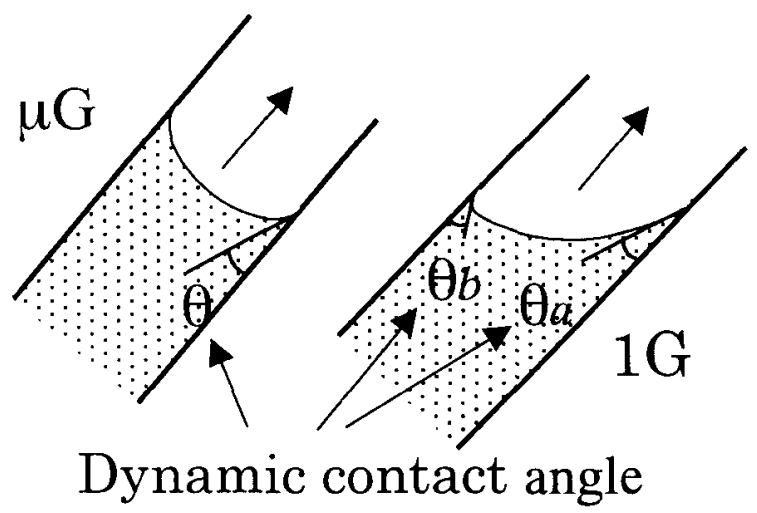

Fig. 6 The dynamic contact angle in the inclined tube under $\mu \mathrm{G}$ and $1 \mathrm{G}$ condition.

The experimental capillary flow rate shown in the Fig.4 is slightly smaller than the calculated result. This slight difference might be explained by introducing the non-zero dynamic contact angle of about $35^{\circ}$.

The calculated capillary rises with varying the contact angle under normal gravity condition bases on the eq. (9) are shown in Fig.7 with the experimental values. From this Fig.7, it is found that the result of the experimental capillary rise agrees well with that with $\cos \theta$ of 0.4 , or the dynamic contact angle of $66^{\circ}$. Namely, the behavior of the capillary rise under normal condition might be explained by eq. (9) by introducing the appropriate dynamic contact angle. At this moment, however, it is very difficult to measure the dynamic contact angle in the porous media. To establish the detailed capillary rise mechanism in the porous media, further quantitative work is certainly necessary.

\section{Concluding Remarks}

To investigate the capillary flow phenomena, the capillary flow rate of ethanol through the porous glass plate (average pore diameter is $0.1 \mathrm{~mm}$ ) under microgravity was measured by using the drop-shaft type microgravity facility of JAMIC. Based on the simple model, the rate of capillary rise rate of liquids is developed and is expressed by

$$
\text { where } h_{0}=2 \sigma \cos \theta / \mathrm{a} \rho \mathrm{g} \text {. }
$$

$$
\mathrm{t}=\left(8 \mu / \mathrm{a}^{2} \rho g\right)\left(\mathrm{h}_{0} \ln \left(\mathrm{h}_{0} /\left(\mathrm{h}_{0}-\mathrm{h}\right)\right)-\mathrm{h}\right)
$$

The behavior of the capillary rise under microgravity is reasonably explained by the developed equation. But, the capillary rise along the vertical direction under normal gravity did not agree with the equations, and this disagreement may be explained based on the difference

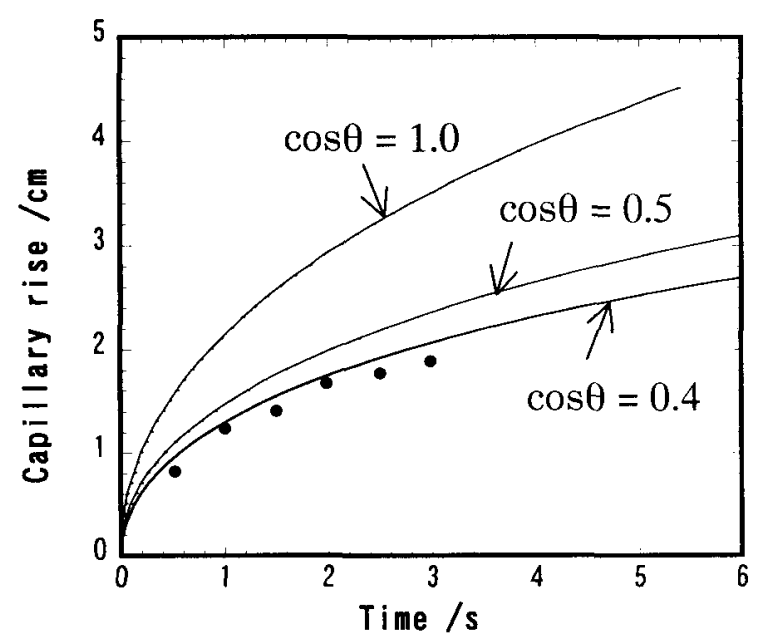

Fig. 7 The capillary rise rate under normal gravity with varying the dynamic contact angles, $\cos \theta$.

between the static contact angle and the dynamic contact angle. By introducing the dynamic contact angle of $35^{\circ}$ for $\mu \mathrm{G}$ and $66^{\circ}$ for $1 \mathrm{G}$, the experimental results are well represented. Taking into account the irregular shape of connected pores in the porous glass plate, the dynamic contact angle of $35^{\circ}$ under $\mu \mathrm{G}$ and $66^{\circ}$ under $1 \mathrm{G}$ may be acceptable.

\section{REFERENCES}

1) R. J. Hansen and T. Y. Toong : J. Colloid Interface Sci., 37 (1971), 196-207.

2) R. L. Hoffman : J. Colloid Interface Sci., 50 (1975), 228-41.

3) E. Rillerts and P. Joos : Chem. Eng. Sci., 35 (1980), 883-87.

4) Y. Shikhmurzaev : AIChE Journal, 42 (1996), 601-12.

5) K. Ishimi, J. Mohri, H. Mukouyama and H. Ishikawa : J. Chem. Eng. Japan, 31 (1998), 914-21.

6) M. Iguchi, M. Ohmi and F. Akao : Nihon Kikai Gakkai Ronnbunnshi, 51 (1990), 436-44. (In Japanese).

7) V. G. Levich : Physicochemical Hydrodynamics, Prentice-Hall, Inc. Englewood Cliffs, N. J., 1962, 382.

8) G. W. C. Kate and T. H. Laby : Tables of Physical and Chemical Constants, Longman Group, New York, (1995), 52.

9) Y.Kanaki, K.Ishii, Y.Sasaki and M.Iguch: Hokkaido University, unpublished research, 1999. 